\title{
Retracted: The Rational Third-Kind Chebyshev Pseudospectral Method for the Solution of the Thomas-Fermi Equation over Infinite Interval
}

\author{
Mathematical Problems in Engineering \\ Received 6 April 2015; Accepted 6 April 2015 \\ Copyright (C) 2015 Mathematical Problems in Engineering. This is an open access article distributed under the Creative Commons \\ Attribution License, which permits unrestricted use, distribution, and reproduction in any medium, provided the original work is \\ properly cited.
}

The paper titled "The Rational Third-Kind Chebyshev Pseudospectral Method for the Solution of the Thomas-Fermi Equation over Infinite Interval" [1] has been retracted as it was found to contain a substantial amount of material, without referencing, from the following published papers: "On the rational second kind Chebyshev pseudospectral method for the solution of the Thomas-Fermi equation over an infinite interval," published in Journal of Computational and Applied Mathematics (Volume 257, February 2014, Pages 79-85), and "Rational Chebyshev pseudospectral approach for solving Thomas-Fermi equation," published in Physics Letters A (Volume 373, Issue 2, 5 January 2009, Pages 210-213).

\section{References}

[1] M. Tavassoli Kajani, A. Kılıçman, and M. Maleki, “The rational third-kind Chebyshev pseudospectral method for the solution of the Thomas-Fermi equation over infinite interval," Mathematical Problems in Engineering, vol. 2013, Article ID 537810, 6 pages, 2013. 